\title{
Small bowel obstruction in an elderly woman
}

\author{
I G Finlay, A Moon, A R Dixon
}

A 78 year old woman presented with a four day history of nausea, intermittent bouts of vomiting, and vague abdominal pain. She had been constipated for three days but was passing flatus. She had no history of abdominal surgery.

On examination she was apyrexial, but clinically dehydrated. Abdominal examination revealed distension with paraumbilical and right upper quadrant tenderness but no associated peritonism, bowel sound were initially active but soon diminished, and there was no palpable hernia. She had a white cell count of $11.4 \times 10^{9} / 1$, haemoglobin of $166 \mathrm{~g} / \mathrm{l}$, and a platelet count of $508 \times 10^{9} / 1$. Urea and

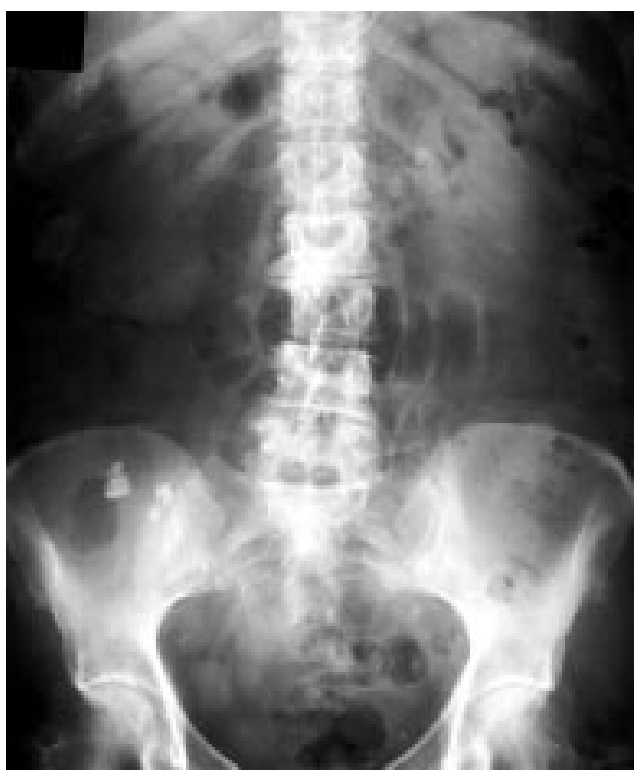

Figure 1 Plain abdominal radiograph of the patient on admission. electrolyte concentrations and liver function tests were unremarkable.

A plain abdominal film was taken which revealed the diagnosis (figs 1 and 2).

\section{Questions}

(1) What was the diagnosis, and how common is the condition in this age group?

(2) What radiological features typical of this condition are present in figs 1 and 2, and which are not?

(3) What treatment is indicated, what important feature of the condition must not be overlooked, and are there any controversial options to be considered?

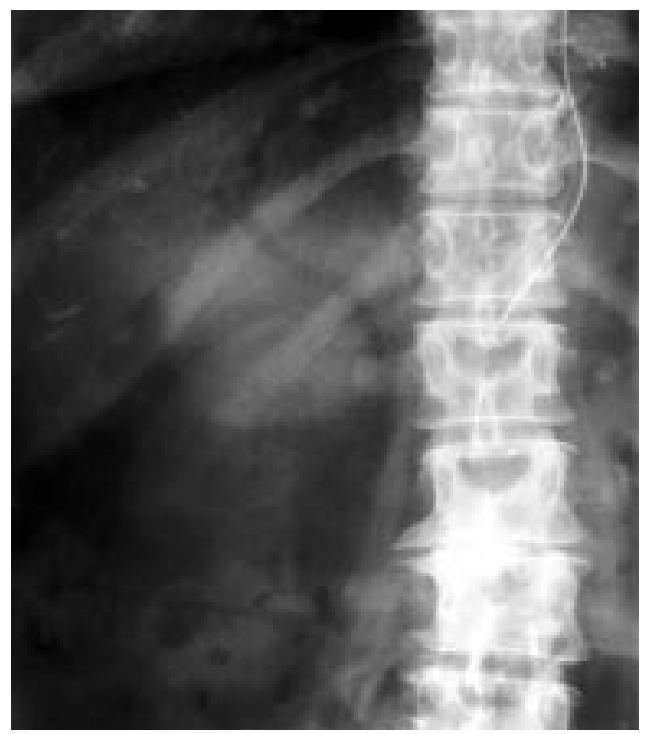

Figure 2 Close up of the right upper quadrant of fig 1.
Department of

General Surgery,

Frenchay Hospital, Bristol, UK

I G Finlay

A Moon

A R Dixon

Correspondence to:

Mr I G Finlay, Department of General Surgery, Ward 9, Gloucester Royal Hospital, Great Western Road, Gloucester GL1 3NN, UK ifinlay@hotmail.com

Submitted 1 December 2000 Accepted 18 December 2000 


\section{Answers}

QUESTION 1

The diagnosis was gallstone ileus, a mechanical intestinal obstruction caused by the impaction of one or more gallstones, having passed into the bowel through a cholecystoduodenal fistula. This presentation is typically vague with three or four days of abdominal pain and vomiting caused by intermittent partial obstruction as the stone "tumbles" distally along the small bowel, eventually impacting as the calibre narrows. Typically impaction occurs in the terminal ileum but can occur anywhere from stomach to rectum.

While the incidence of this condition is relatively low, it is a disease of the elderly (mean age at presentation 72 years ${ }^{1}$ ) and its incidence is consequently rising. More importantly, gallstone ileus is responsible for $25 \%$ of all non-strangulated small bowel obstructions in the elderly, ${ }^{1}$ and should therefore always be excluded when presented with an elderly patient with a suggestive history. As with all gallstone related conditions, there is a female predominance.

\section{QUESTION 2}

The classical radiological features of gallstone ileus were described by Rigler et al in $1941,{ }^{2}$ and are summarised in box 1 .

Intestinal obstruction is clearly seen in fig 1 , as is pneumobilia which is highlighted in fig 2 with the common, right and left bile ducts being clearly demonstrated. No stone is visible, nor were any previously documented. These Rigler criteria are rarely all seen in any one patient, with only $46 \%$ of patients presenting with two or more, ${ }^{3}$ and their absence does not exclude the diagnosis.

An additional feature on plain abdominal $x$ ray films has been described: the appearance of two adjacent fluid levels in the right upper quadrant (Balthazar's sign), the levels lying in the duodenum and adjacent gallbladder.

While plain abdominal radiography remains the principal investigation, some authors advocate the use of computed tomography. In addition to all the features seen on plain radiography, abnormalities in the region of the gallbladder and uncalcified gallstones can be demonstrated.

\section{Box 1: Radiological features of gallstone ileus on plain abdominal films}

- Air (pneumobilia) or contrast medium in the biliary tree.

- Direct or indirect (using contrast medium) visualisation of a stone in the intestine.

- A change in the position of a previously observed stone.

- Evidence of partial or complete intestinal obstruction.
Box 2: Learning points

- Gallstone ileus accounts for $25 \%$ of all non-strangulated bowel obstructions in those over 65 .

- Radiographic features may be diagnostic, but are not always present.

- Laparotomy and enterolithotomy is indicated, with a careful search of the bowel for additional stones being mandatory.

- Concomitant cholecystectomy and fistula closure is not indicated in the majority of patients.

QUESTION 3

There is no role for non-operative intervention; thorough resuscitation and subsequent operation is indicated. At laparotomy the site of obstruction is easily identified and the impacted gallstone palpated. In most cases simple proximal longitudinal enterotomy and stone extraction are all that is required to relieve the obstruction, the enterotomy being closed transversely to avoid narrowing the lumen.

Multiple stones have been reported in 3\% to $40 \%$ of cases of gallstone ileus. ${ }^{3}$ Therefore one should always carefully palpate the gastrointestinal tract in order to detect and remove any second stone that may otherwise cause a prompt recurrence of the obstruction.

There is longstanding controversy regarding management of the cholecystoduodenal fistula. Two questions rise: Should the fistula and gallbladder be excised or not, and if so should this be performed at the time of initial laparotomy, or as a delayed second procedure? No prospective randomised trial exists, or is likely to be performed, the literature consisting of retrospective series, many of which address this issue.

Advocates of concomitant cholecystectomy and fistula closure quote late complication rates of up to $30 \%$ when enterotomy alone is performed. ${ }^{4}$ Complications include symptomatic cholecystitis, cholangitis, and an increased risk of cholangiocarcinoma. However, in most large series the incidence of complications is lower, and it would appear that most fistulae close spontaneously. ${ }^{4}$ Prolonged anaesthesia is best avoided in this elderly population with concomitant medical problems. Dissection of the inflammatory mass surrounding the fistula site is difficult and can prolong the operation by over an hour. Recent larger series have reported slightly increased morbidity and mortality rates associated with concomitant excision of the fistula. ${ }^{15}$ Certainly one stage cholecystectomy and fistula excision is not mandatory, and should only be performed in a fit patient with amenable disease. A second stage procedure would only be considered for fit patients with symptomatic complications.

\section{Final diagnosis}

Gallstone ileus.

1 Reisner RM, Cohen JR. Gallstone ileus: a review of 1001 reported cases. Am Surg 1994;60:441-6.

2 Rigler LG, Borman CN, Noble JF. Gallstone obstruction: pathogenesis and roentgen manifestations. $¥ A M A$ 1941; pathogenesis 
3 Clavien PA, Richon J, Burgan S, et al. Gallstone ileus. Br 7

Surg 1990;77:737-42.
4 Day EA, Marks C. Gallstone ileus: review of the literature and presentation of thirty four new cases. Am $\mathcal{F}$ Surg 1975; 129:552-8.
5 Rodrígues-Sanjuán JC, Casado F, Fernández MJ, et al. Cholecystectomy and fistula closure versus enterolithotomy alone in gallstone ileus. Br f Surg 1997;82(suppl 1):634-7. 